\title{
Zigzag Coverage Scheme Algorithm \& Analysis for Wireless Sensor Networks
}

\author{
Ammar Hawbani \\ School of Computer Science and Technology, University of Science and Technology of \\ China, E-mail: ammar12@mail.ustc.edu.cn \\ Xingfu Wang \\ School of Computer Science and Technology, University of Science and Technology of \\ China, E-mail: Wangxfu@ustc.edu.cn
}

Received: December 7, 2013 Accepted: December 27, 2013 Published: December 31, 2013

DOI: 10.5296/npa.v5i4.4688

URL: http://dx.doi.org/10.5296/npa.v5i4.4688

\begin{abstract}
The problem of having sufficient coverage is an essential issue in wireless sensor network (WSN). A high coverage rate delivers a higher quality of service. The aim of coverage problem is to ensure a minimum number of nodes (at least one sensor) with little redundant data cover every point inside the interest area. In this paper, we provided an algorithm for WSN coverage based on Zigzag Pattern. The interest area divided into multiple Zigzag Patterns with multiple corners and lines segments, each node is deployed in a corner of Zigzag Pattern. Zigzag Pattern Scheme Deployment Algorithm expresses a very high coverage efficiency $91 \%$, as well as, it expands and covers the whole interest area with minimum number of nodes, while it generates a very little coverage redundancy. We provided geometrical analysis to illustrate when the algorithm reaches the maximal and optimal coverage efficiency. The algorithm reaches the maximal and optimal coverage efficiency when the circumference of sensing range for each node is equal to the sum of its vertical Arcs length and horizontal Arcs length, while the optimal length of each line segment in Zigzag Pattern is $\approx \sqrt{3} r$ and the optimal angle of each corner is $60^{\circ}$.
\end{abstract}

Keywords: WSN, WSN Coverage, Grid Coverage, Zigzag Coverage, Coverage Efficiency, Maximum Coverage. 


\section{Introduction}

WSN is a collection of nodes, which are low-cost, low power and small size, carrying out the task of sensing, performing simple data processing and communicating wirelessly over a short distance [1]. Each node contains three main subsystems: the sensing subsystem contains one or more physical sensor devices and one or more analog-to-digital converters as well as the multiplexing mechanism to share them. The processor subsystem executes instructions pertaining to sensing, communication, and self-organization [2]. The communication subsystem contains the transmitter and receiver for sending or receiving information. The sensed data by the sensor devices is generally highly important, and may be of technical or planned Importance. Therefore, one of the primary issues that occur naturally in sensor networks is coverage (how to cover the whole interest field). The good coverage strategy provides a good way to evaluate the performance of whole network [3].

WSNs like other distributed systems, it subjects to a variety of unique constraints and challenges such as restricted sensing and communication ranges as well as limited battery capacity [3]. These challenges affect the design of a WSN [2], and bring issues such as coverage, connectivity, network lifetime, self-managing and data aggregation [4] [5]. Good coverage scheme helps a lot in getting the desired purpose of the network using the minimum economic costs; not only this, but also the data routing and transferring depending mainly on the sensors deployment in interest workplace.

The rest of this paper is structured as: Section 2, related works are overviewed. Section 3 describes a modulation for the problem. In section 4, we will provide a zigzag pattern deployment details and algorithm as well as we compute the maximum coverage obtains by this scheme. An evaluation was provided from the point of view of energy consumption, deployment space and communications messages in section 5.

\section{Related work}

Many of coverage schemes have been proposed such as target coverage. target coverage means to monitor a number of fixed targets, this type of coverage scheme mentioned in [6] has noticeable military applications, the authors extensive tests to not only detect targets, but to classify and track them , also the authors in [7] tried to detect targets while conserving energy. Barrier coverage scheme discusses the detection of movement across a barrier of node; this scheme explored in the reference [8] with details.

Most of researches assumed the nodes are static and stuck in their locations once they deployed. However, newer nodes are able to relocate themselves dynamically after deployment. The coverage of mobile nodes is more complex than static ones [3] because they need a dynamic geographic computation to get their location updates whenever detected a necessity to maximize the coverage. In the algorithm [9], each node has the ability to move in order to provide maximum coverage. In [10], Zou provides a combination of attractive and repulsive forces to determine virtual motion paths and the rate of movement for the randomly placed sensors. [11] Comes with moving energy consumption improvement of algorithm 


\section{1) Macrothink Mnstitute}

proposed in [10]. Two range-free, distributed and probabilistic mobile beacon-assisted localization approaches for static WSNs, MBL and A-MBL is proposed in [12].

\section{Problem Modulation}

Work area $W_{n}$ :The interest area to be covered by nodes it is a set of point 2D space.

Set of sensors $S_{k}$ : The sensors to be deployed in the working area. Each one has well know location. The aim of the coverage is to ensure that every point

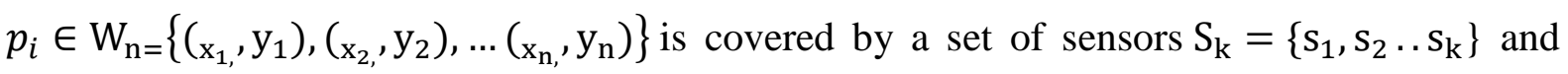
satisfied the following constraints:

1. Connectivity: There is at least 1 path $\mathrm{s}_{\mathrm{i}} \mathrm{s}_{\mathrm{i}+1} \mathrm{~s}_{\mathrm{i}+2} \ldots \mathrm{B}_{\mathrm{s}} ; \mathrm{s}_{\mathrm{i}+\mathrm{j}} \in \mathrm{S}_{\mathrm{k}}, \mathrm{i}, \mathrm{j}>1, i+j<k+1$ from the current node $s_{i}$ to the base station $B_{s}$.

2. Minimum nodes: $\mathrm{k}$ is the minimum number of nodes; get the maximum coverage area with the minimum number of nodes.

3. Less redundant data: the larger the overlapped area between the sensors in the network the more amounts of redundant data to generated and more power to consumed and busier the network will be.

\section{Definition 1:}

We can calculate the Node Coverage Redundancy with reference to the sum of the intersection areas within overlapped sensors (the area would be covered by more than one sensor at onetime) between adjacent nodes.

\section{Assumption 1:}

All the sensors inside the network are Homogeneous, that is to say, they have the same sensing range and communication range, which can monitor the whole direction around, and we say the coverage is $r$ radius of a circular area A. $A=\pi r^{2}$ [13].

\section{Assumption 2:}

The sensors of the network have the same transmission power, that is to say, they have the same ability to detect the target when it moves within sensing range.

\section{Definition 2:}

Calculation of maximum effective of area coverage of the whole network is the effective coverage area of all node inside the network it can be calculated as below [13].

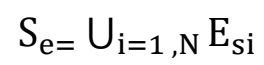

\section{Definition 3:}

Calculation of Coverage Efficiency of the whole network is the ratio of the union of (in 


\section{Macrothink

the definition 2) effective coverage area of all the nodes and their sum. Coverage efficiency is determined using the following equation [13].

\section{Zigzag Pattern Deployment}

\subsection{Motivation}

A zigzag is a pattern made up of small corners at variable angles, though constant within the zigzag, tracing a path between two parallel lines; it can be described as both jagged and regular. In Zigzag Pattern Deployment Scheme, we will assume that the small corners with the same angle, and denote to the corner angle by $\theta$. Each Zigzag Pattern consist of finite number of lines segments and corners. Here we denote to each Zigzag Pattern by:

$$
\mathrm{Z}_{i}=\left\{l_{0}, l_{1}, l_{2} \ldots l_{i}\right\}
$$

The $\left|\mathrm{Z}_{i}\right|$ is denote to the number of lines segments. $l_{j}$ is a line segment where $i \geq j \geq 0$. The length of line segment denoted by $\left|l_{i}\right|$.

Alternatively, the corners can express the Zigzag Pattern as:

$$
\mathrm{Z}_{i}=\left\{c_{0}, c_{1}, c_{2} \ldots c_{m}\right\}
$$

where $c_{j}$ is a corner between two intersected lines. The distance between two corners is denoted by $D$.

Moreover, we suppose that each sensor $\mathrm{s}_{i}$ knows its location well, $P_{i} \in \mathrm{W}_{\mathrm{n}}$. To ensure full coverage of each point inside the interest area, we will divided it - interest area - into multiple separated Zigzag Patterns, that is to say:

$$
W_{z}=\left\{Z_{0}, Z_{1}, Z_{2} \ldots Z_{z}\right\}
$$

The symbol $\left|\mathrm{W}_{\mathrm{z}}\right|$ is denoted to the number of Zigzag Patterns to be placed in the interest area.

\subsection{Zigzag Pattern Deployment Algorithm}

The Zigzag algorithm starts by divide the interest area into $\left|\mathrm{W}_{\mathrm{z}}\right|$ Zigzag Patterns, the number of Zigzag Patterns is $\left|\mathrm{W}_{\mathrm{z}}\right|=\left(\mathrm{h} / l_{i} / 2\right)$, where $\mathrm{h}$ is the length of interest area and $l_{i}=2 r \times c, 0.1 \geq c \geq 0.99$. $c$ is the Percentage of vertical overlapping between the centers of two sensors deployed on the same Zigzag Pattern. The number of lines segments in 
the pattern $\left|Z_{i}\right|=\mathrm{w} / \mathrm{D}$, where $\mathrm{w}$ is the width of interest area and $\mathrm{D}=\mathrm{r} / 2 \times v, 0.1 \geq$ $v \geq 0.99$. Here $v$ is the Percentage of horizontal overlapping between the centers of two adjacent sensors deployed horizontally in the same Zigzag Pattern. See Figure 1.

The Sensors distributed along the intersection corners of the lines of each Zigzag Pattern $\mathrm{Z}_{i}=\left\{c_{0}, c_{1}, c_{2} \ldots c_{m}\right\}$. Each corner becomes a center point of a node, and each corner is either to be a bottom pointed $c_{0}, c_{2}, c_{4} \ldots$ or to be a top pointed $c_{1}, c_{3}, c_{5} \ldots$ (see Figure 1 ). For these bottom pointed corners, we called by the first level of Zigzag Pattern. In other hand, the top pointed corners are called by the second level.

Figure 1: Zigzag Pattern description

Every node will be located either in the first level or in the second level. The sensor $k$ located on the line segment $m$ at the Zigzag Pattern $i$ is denoted by $s_{k}^{\left(\mathrm{Z}_{i}, c_{m}\right)}$; its location can be determined by equation (2).

$$
s_{k}^{\left(\mathrm{Z}_{i}, c_{m}\right)}=\left\{\begin{array}{c}
p\left(m \times D, l_{m} \times(\text { level }-1)\right) \text { where level }=2 i+1 \\
p\left(m \times \mathrm{D}, l_{m} \times(\text { level })\right) \text { where level }=2 i+1
\end{array}\right.
$$

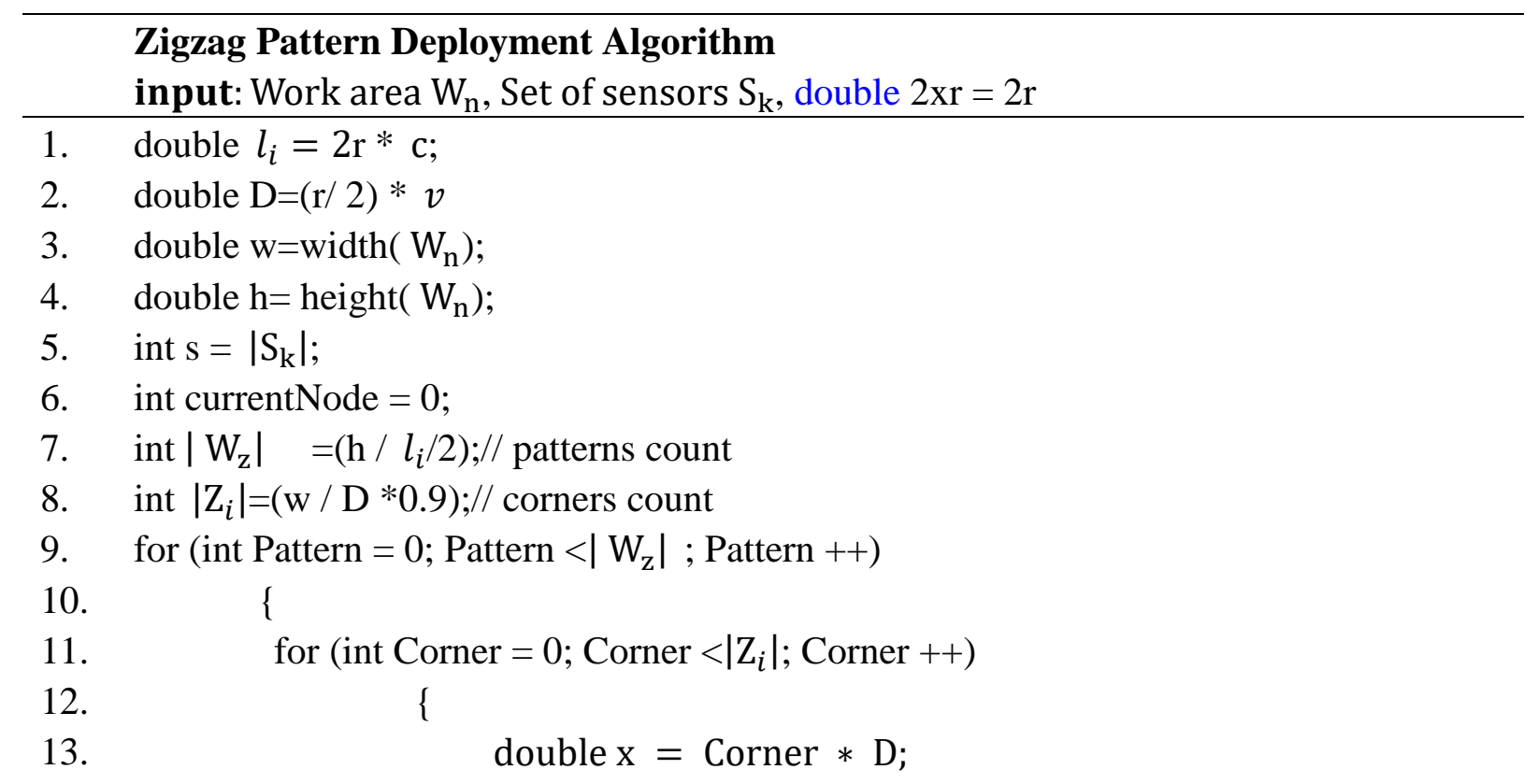


14.

15.

16.

17.

18.

19.

20.

21.

22.

23.

24.

25.

26.

27.

28.

29.

30.

31.

32.

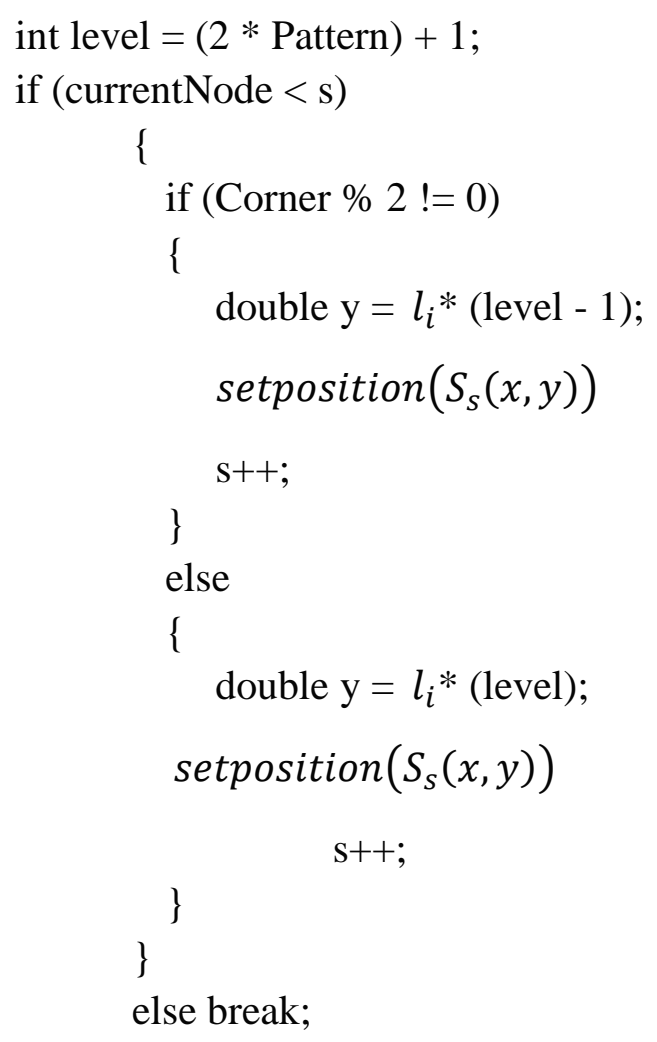

\subsection{Notes for Maximizing the coverage efficiency}

The Zigzag Pattern Deployment Algorithm is controlled by two parameters. The percentage of horizontal overlapping $v$ and the Percentage of vertical overlapping $c$. In the Figure 2, the effective of $v$ and $c$ parameters is illustrated. If the value of $v$ is small, then the value of $\mathrm{D}$ will be bigger which lead the greater value of $\theta$. When the angle $\theta$ gets greater, that leads to bigger distance between the lines segments and loss the coverage of some points. See Figure 3. The greater the value of $\boldsymbol{v}$ the smaller the distance between the corners (located at the same level) of Zigzag Pattern. Hence the value of $\boldsymbol{\theta}$ will be smaller, this leads to more coverage redundancy and affects the network lifetime and performance.

In other hand, as long as the value $c$ gets bigger, the length of each line segment in the Zigzag Pattern will be smaller which might leads to maximize the coverage redundancy. The coverage redundancy increasing if the distance between the corners is smaller. When this value get smaller, this might lead to loss the full coverage of sensing field.

The percentage of vertical overlapping c effects the $\left|\boldsymbol{l}_{\boldsymbol{i}}\right|$ of lines segments, and the Percentage of vertical overlapping $\boldsymbol{v}$ effect the size of measured angle $\boldsymbol{\theta}$. In next subsection, we will explain the optimal values for $\boldsymbol{\theta} \&\left|\boldsymbol{l}_{\boldsymbol{i}}\right|$ which they can ensure the full coverage and maximum coverage efficiency. 


\section{Macrothink Institute ${ }^{\mathrm{m}}$}

Table 1: illustration of Fig.2

\begin{tabular}{|l|l|l|l|c|c|}
\hline & v & c & $\begin{array}{l}\text { Coverage } \\
\text { Redundancy }\end{array}$ & Full Coverage & $\begin{array}{l}\text { Maximum } \\
\text { Coverage }\end{array}$ \\
\hline (a) & 0.99 & 0.99 & No & No & No \\
\hline (b) & 0.50 & 0.50 & Yes (more) & Yes & No \\
\hline (c) & 0.70 & 0.70 & Yes ( more ) & Yes & No \\
\hline (d) & 0.88 & 0.76 & Yes (less ) & Yes & Yes \\
\hline
\end{tabular}

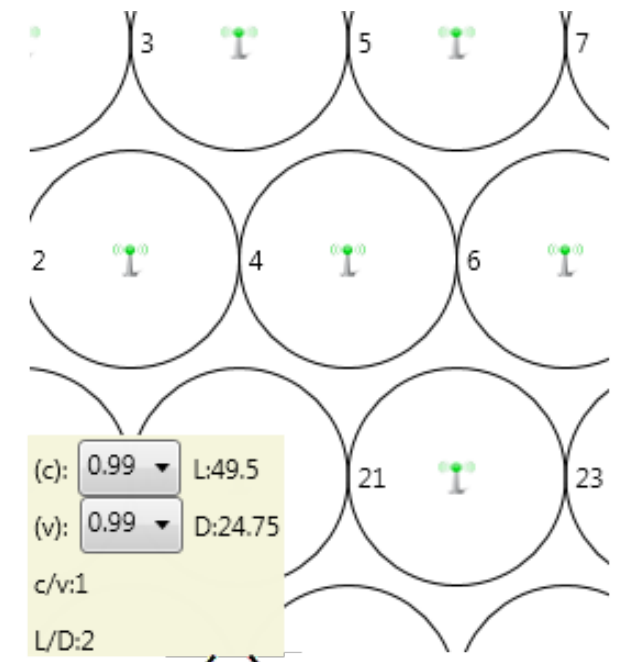

(a)

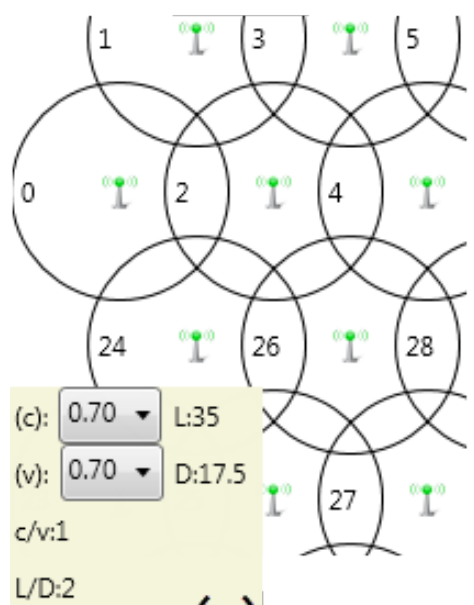

(c)

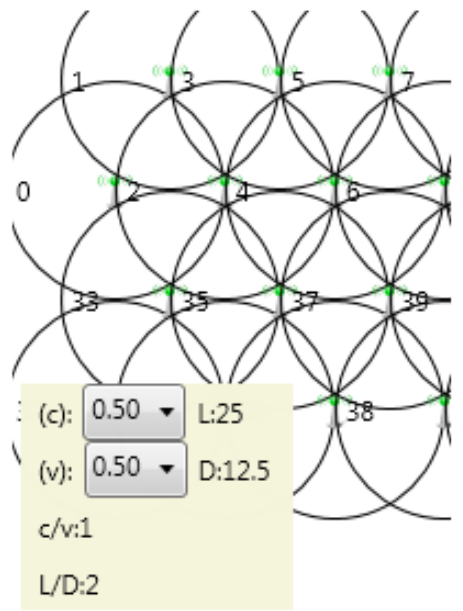

(b)

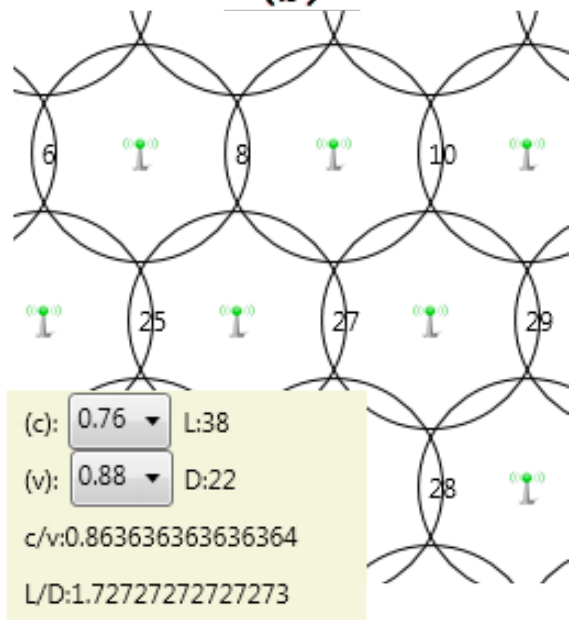

(d)

Figure 2: Zigzag Pattern Deployment with different horizontal \& vertical overlapping percentage

The slope of each line segment in the Zigzag Pattern is $\pm \frac{2 c}{v}$. In addition, we can find the angle $\theta$ by the equation (3).

$$
\theta=\tan ^{-1}\left(\frac{\left(\frac{2 c}{v}+\frac{2 c}{v}\right)}{\left(1+\left(\frac{2 c}{v}\right)^{2}\right)}\right)
$$



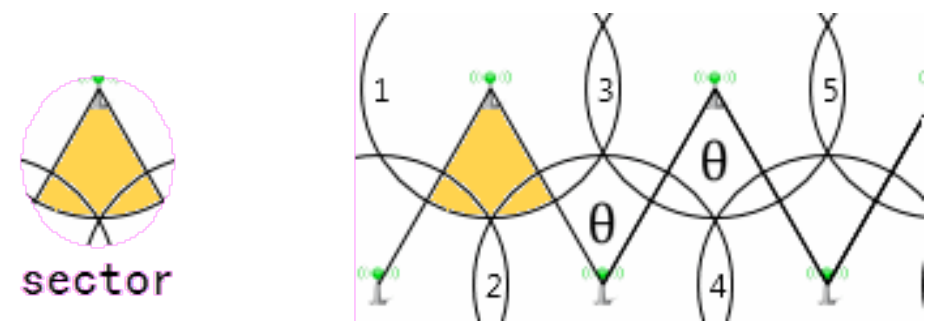

Figure 3: Angle of Zigzag Pattern

\subsection{Finding The Optimal $\boldsymbol{\theta}$}

The angle $\boldsymbol{\theta}$ between any two touchlines segments (the value of corner) and the length of line segment $\left|\boldsymbol{l}_{\boldsymbol{i}}\right|$ effects the maximum coverage and coverage efficiency of Zigzag Pattern. In this subsection, we will find the optimal angle of corners and the optimal length of line segment.

We will start by define the two chords, the vertical and diagonal chords. As shown in Figure 4. The lengths of vertical and diagonal chords are effected by the vertical and horizontal overlapping percentage $(v \& c)$. The lengths of two cords must be within the $1 \sim 2 r$. The optimal coverage efficiency obtains when:

1- Lengths of vertical and diagonal chords are the same.

2- The vertical and diagonal chords have no intersection.

The smaller the length of vertical and diagonal chords is, the more the uncovered points will be. In other hand, the longer the length is, the more the coverage redundancy will be generated.

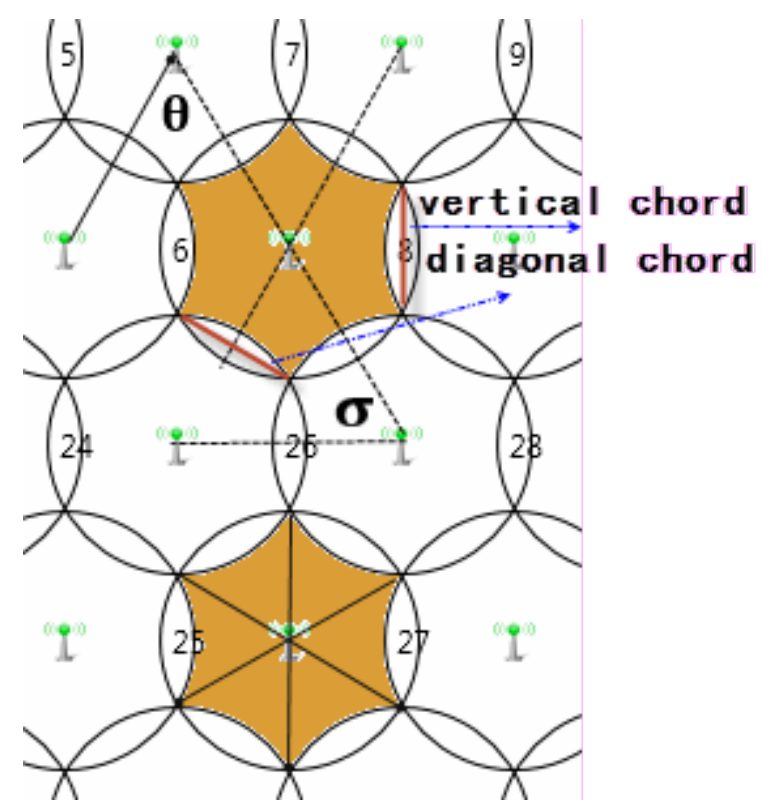

Figure 4: Angle of Zigzag Pattern 


\section{Macrothink

The horizontal angle $\boldsymbol{\sigma}$ is the angle between (D) and adjacent corner in the same level of Zigzag pattern.

\section{Theorem 1:}

The Maximum Coverage and the Optimal Efficiency of Zigzag Pattern Deployment can be achieves only if the angle of corner $\boldsymbol{\theta}$ and horizontal angle $\boldsymbol{\sigma}$ are Equivalence, 60 degree.

\section{Proof:}

In Zigzag Pattern, each node contains similar number of vertical Arcs $\mathrm{V}_{i}$ and horizontal Arcs $\mathrm{H}_{i}$. There are four horizontal Arcs $\mathrm{H}_{i}=\left\{\operatorname{Arc}_{1}, A r C_{2}, A r c_{4}, A r c_{5}\right\}$ and two vertical $\operatorname{Arcs} V_{i}=\left\{A r c_{3}, A r c_{6}\right\}$. See the Figures 5 (a), 5 (b) and 5 (c).

The Circumference of sensing range is:

$$
\mathrm{C}=2 \pi r
$$

The length of each horizontal Arc is

$$
\left|\mathrm{H}_{i}\right|=\mathrm{C} \times \frac{\Theta}{360}
$$

The total length of horizontal Arcs is

$$
|\mathrm{H}|=\sum\left|\mathrm{H}_{i}\right|
$$

The length of each vertical Arc is

$$
\left|V_{i}\right|=C \times \frac{\sigma}{360}
$$

The total length of vertical Arcs is

$$
|\mathrm{V}|=\sum\left|\mathrm{V}_{i}\right|
$$

We can define the Coverage Function by:

$$
\mathrm{CF}=|\mathrm{V}|+|\mathrm{H}|
$$

Which means the Circumference of sensing range for each node is equal to the summation of its vertical Arcs length and horizontal Arcs length. Coverage Function has three cases are shown in equation (4).

$$
\mathrm{CF}= \begin{cases}\text { Maximum } & |\mathrm{V}|+|\mathrm{H}|=C \\ \text { Miss_covered } & |\mathrm{V}|+|\mathrm{H}|<C \\ \text { Redandancy } & |\mathrm{V}|+|\mathrm{H}|>C\end{cases}
$$

The three cases are illustrated in the Figures 5 (a), 5 (b) and 5 (c). In Table 2, Table 3, and Table 4 , the sensing range is $80 \mathrm{px}, \mathrm{C}=2 \pi \mathrm{r}, \mathrm{C}=502.65$ and the length of horizontal chords is calculated by $\mathrm{C} \times \Theta / 360$, while the length of vertical chords is calculated by $\mathrm{C} \times \boldsymbol{\sigma} / 360$. 
Table 2: Arcs Length for nodes shown in figure 5(a)

\begin{tabular}{|c|c|c|c|c|c|c|c|}
\hline & $\Theta$ (Degree) & $\sigma$ (Degree) & $\mathbf{L}$ & D & \multicolumn{2}{|c|}{ Arcs } & Circumference \\
\hline \multirow[t]{7}{*}{ (A) } & \multirow[t]{7}{*}{64.61} & \multirow[t]{7}{*}{57.69} & \multirow[t]{7}{*}{54.4} & \multirow[t]{7}{*}{34.4} & 1 & 90.21 & \multirow[t]{7}{*}{502.65} \\
\hline & & & & & 2 & 90.21 & \\
\hline & & & & & 3 & 80.54 & \\
\hline & & & & & 4 & 90.21 & \\
\hline & & & & & 5 & 90.21 & \\
\hline & & & & & 6 & 80.54 & \\
\hline & & & & & Total & 521.92 & \\
\hline
\end{tabular}

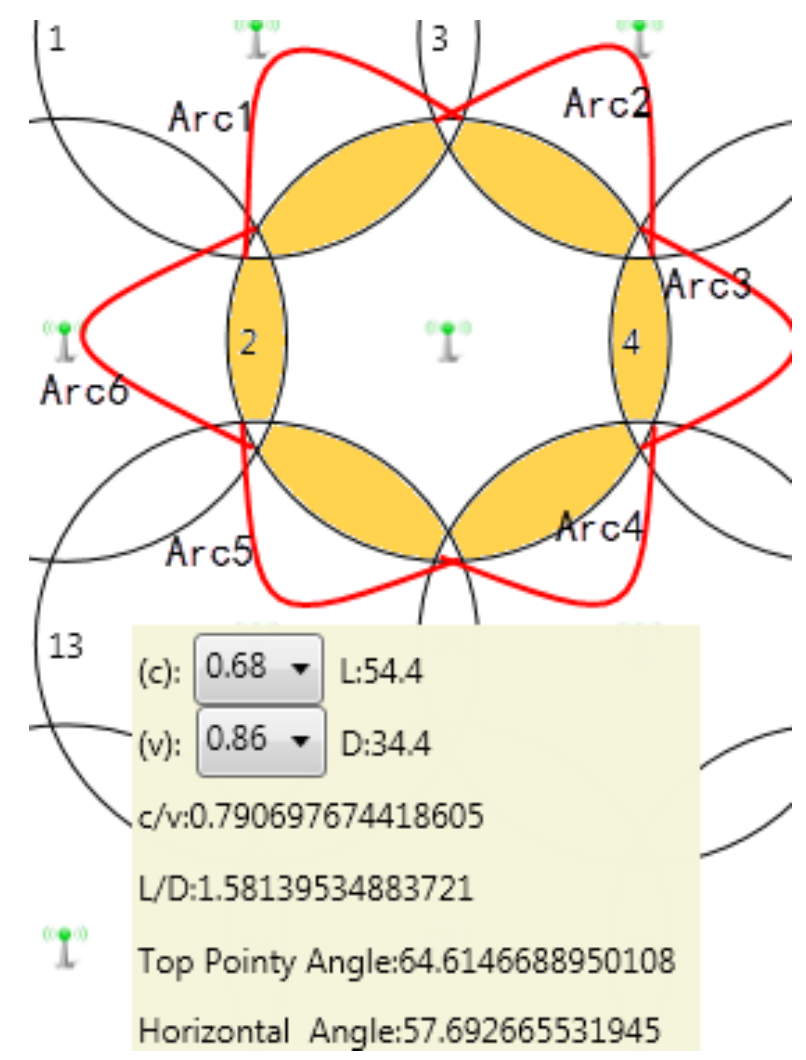

Figure 5 (a) Coverage Redundancy Case

Table 3: Arcs Length for nodes shown in figure 5(b)

\begin{tabular}{|c|c|c|c|c|c|c|c|}
\hline & $\Theta$ (Degree ) & $\alpha$ (Degree) & $\mathbf{L}$ & D & \multicolumn{2}{|c|}{ Arcs } & Circumference \\
\hline \multirow[t]{7}{*}{ (A) } & \multirow[t]{7}{*}{56.51} & \multirow[t]{7}{*}{61.74} & \multirow[t]{7}{*}{64} & \multirow[t]{7}{*}{34.4} & 1 & 78.90 & \multirow[t]{7}{*}{502.65} \\
\hline & & & & & 2 & 78.90 & \\
\hline & & & & & 3 & 86.20 & \\
\hline & & & & & 4 & 78.90 & \\
\hline & & & & & 5 & 78.90 & \\
\hline & & & & & 6 & 86.20 & \\
\hline & & & & & Total & 488 & \\
\hline
\end{tabular}




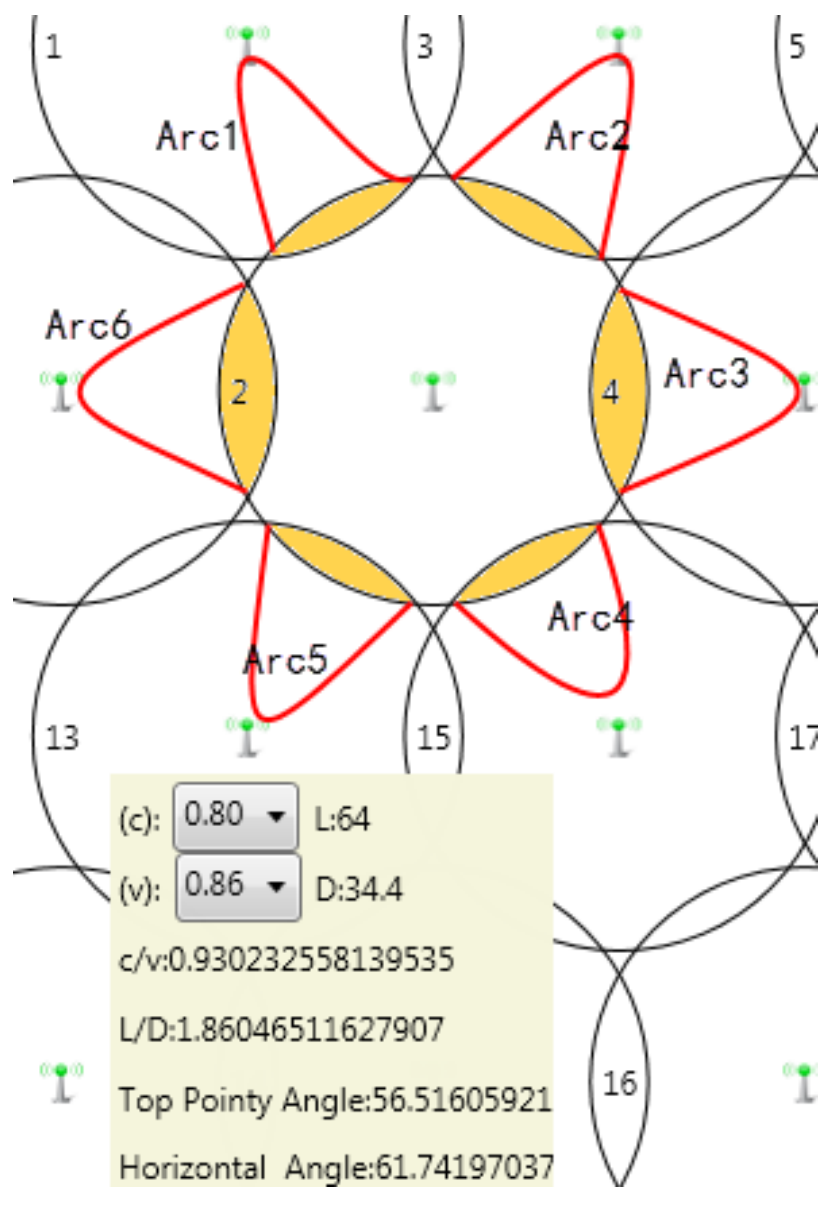

Figure 5(b): Miss-Coverage Case

Table 4: Arcs Length for nodes shown in figure 5(c)

\begin{tabular}{|c|c|c|c|c|c|c|c|}
\hline & $\Theta$ (Degree) & $\alpha$ (Degree) & $\mathbf{L}$ & D & \multicolumn{2}{|c|}{ Arcs } & Circumference \\
\hline \multirow[t]{7}{*}{ (A) } & \multirow[t]{7}{*}{59.65} & \multirow[t]{7}{*}{60.17} & \multirow[t]{7}{*}{60} & \multirow[t]{7}{*}{34.4} & 1 & 83.28 & \multirow[t]{7}{*}{502.65} \\
\hline & & & & & 2 & 83.28 & \\
\hline & & & & & 3 & 84.01 & \\
\hline & & & & & 4 & 83.28 & \\
\hline & & & & & 5 & 83.28 & \\
\hline & & & & & 6 & 84.01 & \\
\hline & & & & & Total & 501.14 & \\
\hline
\end{tabular}




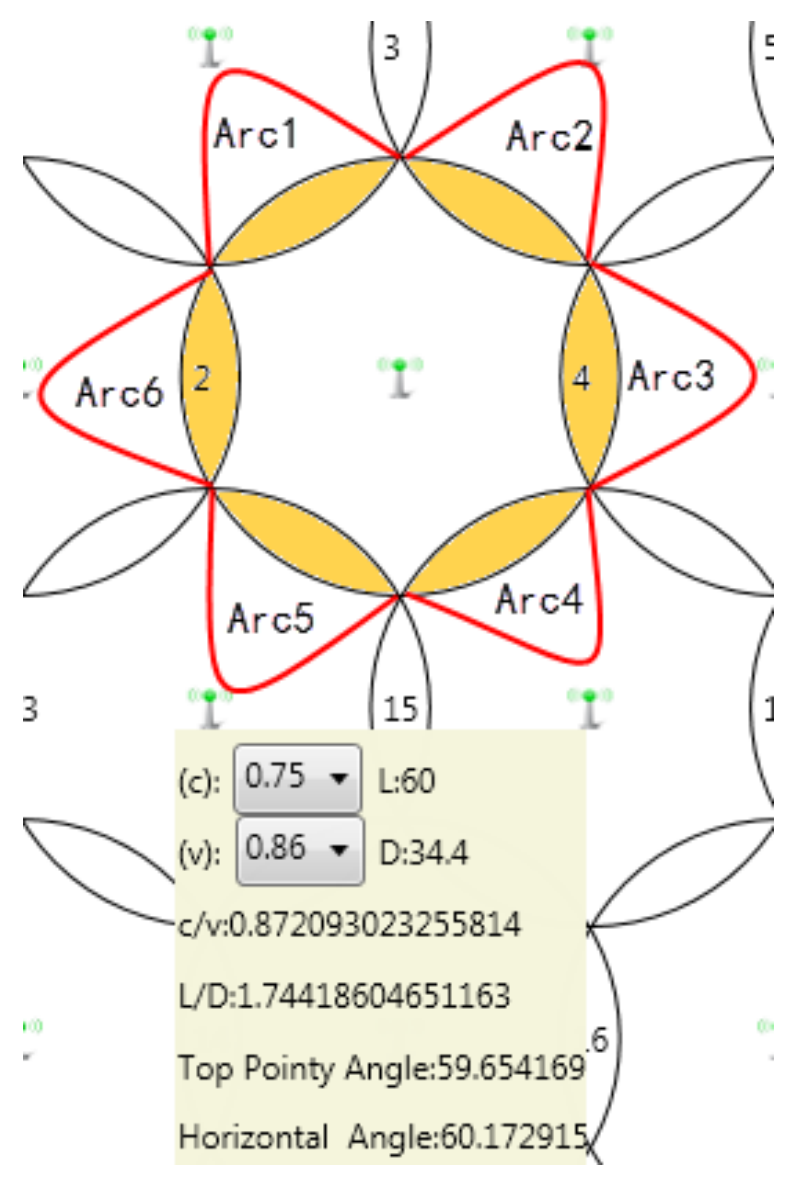

Figure 5(c): Maximum Coverage Case

As clarified, the maximum coverage achieves when the Circumference of sensing range for each node is equal to the summation of its vertical Arcs length and horizontal Arcs length. That is when

$$
\mathrm{C}=|\mathrm{V}|+|\mathrm{H}|
$$

This means the Arcs (six) are the same length, and the two angles $\boldsymbol{\theta} \& \boldsymbol{\sigma}$ are Equivalence.

$$
\boldsymbol{\theta}=\boldsymbol{\sigma}=\frac{360^{\circ}}{6}=60^{\circ}
$$

The angles of both vertical and horizontal chords are the same, this mean they have the same length and they are separated and there is no intersection between them. To explain why there is no intersection between them we suppose we have three overlapped sensors $\left\{s_{1}, s_{2}, s_{3}\right\}$,The intersection between the vertical and horizontal chords laid in $s_{1}$ is impossible when the intersection point between $s_{2}, s_{3}$ is not exactly on the border of $s_{1}$. 


\section{Lemma 1:}

For a set of node $S=\left\{s_{1}, s_{2} \ldots s_{i}\right\}$, say we have $s_{j}$ is a node overlapped with all nodes $s_{1}, s_{2} \ldots s_{i}$, the chords laid in $s_{j}$ are intersect only if any intersection point of any two nodes is located within the sensing range of the node $s_{j}$.

Note: It is easy to prove this lemma if we just measure the total length of arcs located within the sensing range of a node. As long as the total length of arcs is greater than the Circumference of the node, this means there is an intersection between the chords inside the node.

The optimal length of each line segment in Zigzag Pattern is $\approx \sqrt{\mathbf{3}} \boldsymbol{r}$

Table5: Optimal Zigzag line Length for different sensing range

\begin{tabular}{|c|c|}
\hline $\mathbf{r}$ & $\mathbf{L}$ \\
\hline 30 & 51.6 \\
\hline 40 & 68.8 \\
\hline 50 & 86 \\
\hline 60 & 103.2 \\
\hline
\end{tabular}

4.5. The Maximum Coverage and Optimal Coverage Efficiency of Zigzag Pattern Deployment

According to Definition 1, 2, and 3, as well as to equation (1), we will start computing the maximum coverage $A_{t c 1}$ by finding the area of sensing range which covered by two nodes $A t_{c 2}$. The maximum coverage $A_{t c 1}$ is equal to the total area $A_{t}$ subtracting the area covered by two nodes $A_{t c 2}$.

$$
A_{t c 1}=A_{t}-A_{t c 2}
$$

The optimal value of $\boldsymbol{\theta}$ is $\pi / 6$. That is to say, the sensing range of each node is divided into 6 equal sectors. The area of each sector is $\frac{\pi r^{2}}{6}$. As displayed in the Figure (7), the area of sector $A_{\text {sec }}$ is equal to the area of segment $A_{s e g}$ pulse the area of triangle $A_{t r}$.

$$
A_{\text {seg }}=A_{\text {sec }}-A_{\text {tr }}
$$

The triangle is Equilateral, its area is

$$
A_{t r}=\frac{r^{2}}{2}
$$

The area of segment then, is

$$
A_{\text {seg }}=\frac{\pi r^{2}}{6}-\frac{r^{2}}{2}=\frac{\pi r^{2}-3 r^{2}}{6}=\frac{r^{2}(\pi-3)}{6}
$$




\section{Macrothink

For each two-covered region, the area is

$$
A_{c 2}=2 A_{s e g}
$$

Then

$$
A_{c 2}=\frac{r^{2}(\pi-3)}{3}
$$

For each node, the total area of two-covered regions is:

$$
A_{t c 2}=6 A_{c 2}
$$

Then

$$
A_{t c 2}=2 r^{2}(\pi-3)
$$

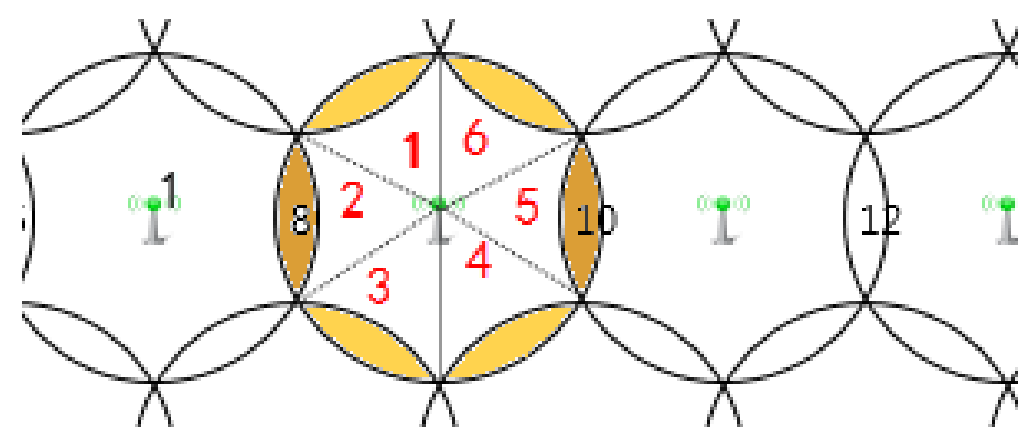

Figure 7: Regions in Zigzag Pattern Node

Using the Assumption 1, the total area of node is

$$
A_{t}=\pi r^{2}
$$

From equations (5), (6) and (7), we can get the Maximum Coverage of Zigzag Patten

$$
A_{t c 1}=A_{t}-A_{t c 2}=\pi r^{2}-2 r^{2}(\pi-3)
$$

The Maximum Coverage Efficiency is

$$
\mathrm{A}_{\max }=\frac{\pi r^{2}-2 r^{2}(\pi-3)}{\pi r^{2}}=91 \%
$$

The Minimum Coverage Redundancy is 


$$
\mathrm{A}_{\min }=\frac{2 r^{2}(\pi-3)}{\pi r^{2}}=9 \%
$$

\section{Performance evaluation}

In this subsection, we will try to evaluate the algorithm from energy consumption, deployment space, communications messages and sensor lifetime points of views. For this purpose, we have develop a special software using c\# visual studio 2012. We have implemented our experiment in $2 D$ space. The work area $896 p x \times 590 p x$.

\subsection{Deployment space}

Considering the cost and economical situations, some users consider the coverage schemes that expands and covers the whole interest area with minimum number of nodes. According to our experiment indicated in the Figure 8 and 9 the Zigzag Pattern Deployment scheme express economical deployment space, which leads to minimize the cost of network. With radius 50, 303 nodes are deployed using Zigzag Pattern Algorithm in Fig8.

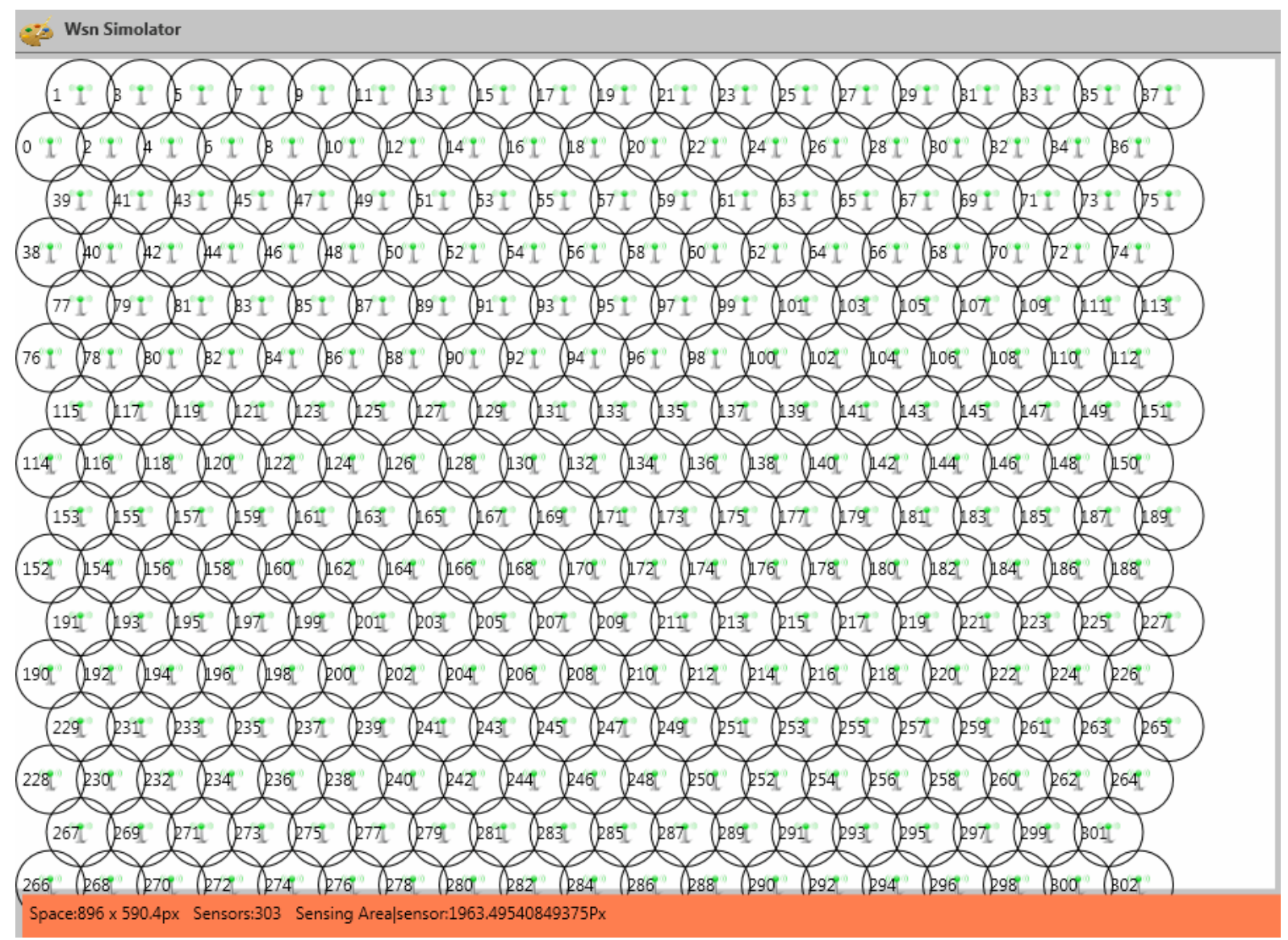

Figure 8: Zigzag Pattern Deployment scheme implementation in 2D space

Our simulation results show that Zigzag Pattern schema save more nodes comparing with 
gird coverage deployment. See Fig9. In table 6, the values listed are showing the results of applying the Zigzag algorithm and Square Grid algorithms. The results shows that the number of nodes required in the case of applying the Zigzag algorithm is less than the number of nodes required when applying Square Grid algorithms by $13 \%$. The sensing radius in this experiment is $10 p x$.

Table 6: Deployment space compression (Zigzag and Square Grid)

\begin{tabular}{|l|c|c|c|}
\hline & Interest Area $\left.\mathbf{( p x}^{\mathbf{2}}\right)$ & Nodes Count (Zigzag Pattern ) & Nodes Count (Square Grid ) \\
\hline 1. & 10000 & 32.28935485 & 32.95143942 \\
\hline 2. & 22500 & 72.65104842 & 74.14073869 \\
\hline 3. & 40000 & 129.1574194 & 139.6489133 \\
\hline 4. & 62500 & 201.8084678 & 218.201427 \\
\hline 5. & 90000 & 290.6041937 & 314.2100548 \\
\hline 6. & 122500 & 395.544597 & 427.6747969 \\
\hline 7. & 160000 & 516.6296777 & 558.5956531 \\
\hline 8. & 202500 & 653.8594358 & 706.9726234 \\
\hline 9. & 302500 & 976.7529843 & 1056.094907 \\
\hline 10. & 360000 & 1162.416775 & 1256.840219 \\
\hline 11. & 422500 & 1364.225243 & 1475.041646 \\
\hline
\end{tabular}

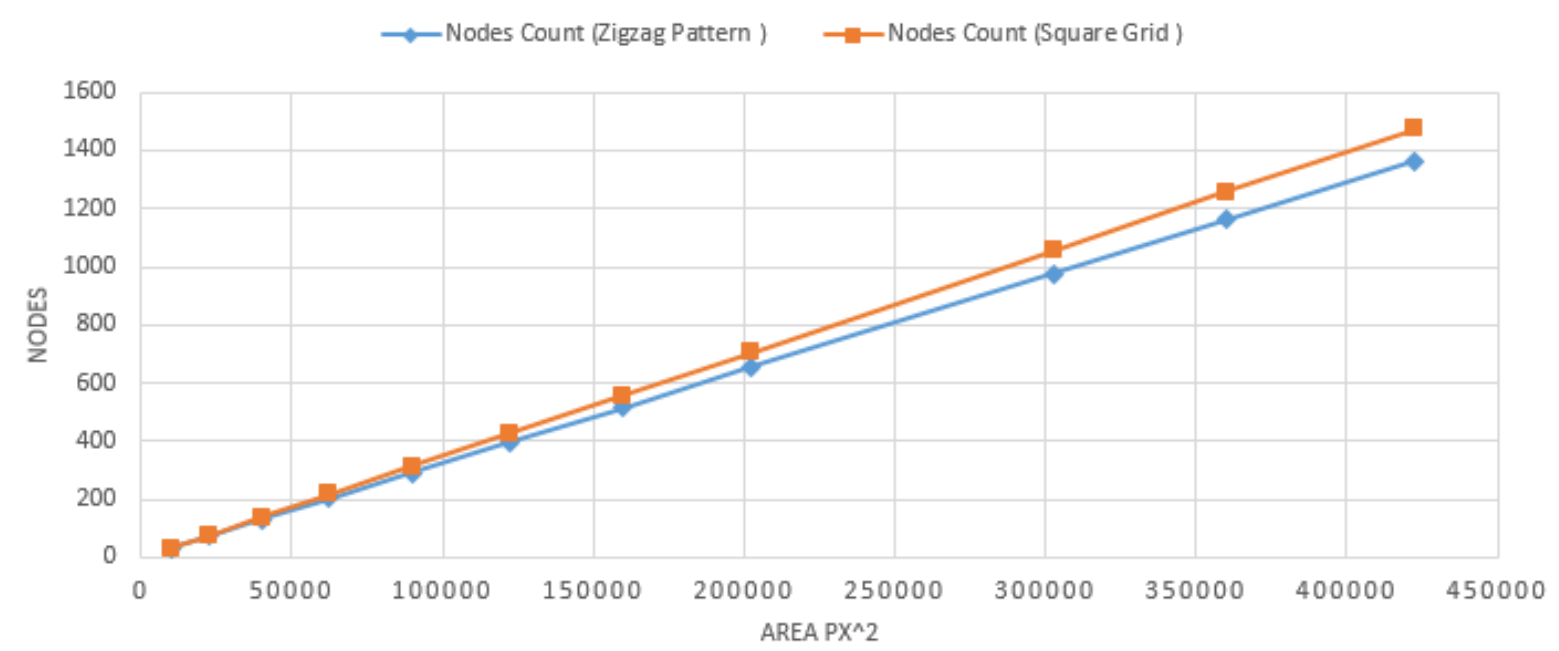

Figure 9: Deployment space compression (Zigzag and square Grid)

\subsection{Coverage and Data Redundant}

According to our experiment, the Zigzag Pattern coverage scheme shows an excellent performance. It can cover a large area with the possible minimum number of node. The area of one-covered regions occupies $91 \%$ of total area. In other hand, the area of two-covered regions is $9 \%$. See the fig 10 and table7. 
Table 7: Zigzag Pattern: Maximum Coverage \& Coverage Redundancy

\begin{tabular}{|c|c|c|c|}
\hline Nodes & Total Area & Maximum Coverage & Coverage Redundancy \\
\hline 1 & 314.1592654 & 285.8849315 & 28.27433388 \\
\hline 5 & 1570.796327 & 1429.424657 & 141.3716694 \\
\hline 50 & 15707.96327 & 14294.24657 & 1413.716694 \\
\hline 100 & 31415.92654 & 28274.33388 & 3141.592654 \\
\hline 200 & 62831.85307 & 57176.9863 & 5654.866776 \\
\hline 300 & 94247.77961 & 85765.47944 & 8482.300165 \\
\hline 400 & 125663.7061 & 114353.9726 & 11309.73355 \\
\hline 500 & 157079.6327 & 142942.4657 & 14137.16694 \\
\hline
\end{tabular}

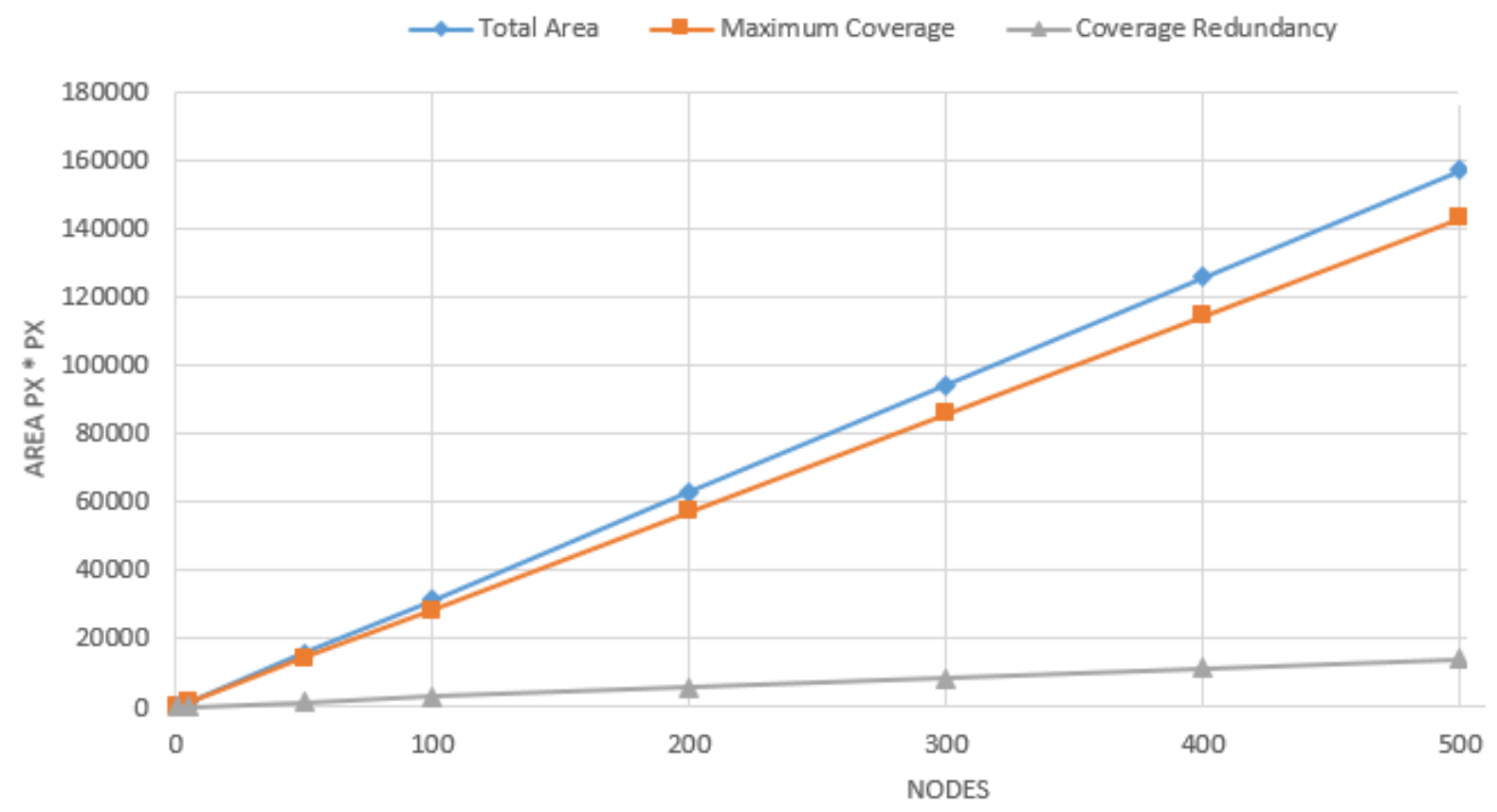

Figure 10: Coverage and Data Redundant

\subsection{Communication and Energy Consumption}

The number of messages depends on the degree of overlapping. The more the degree of coverage is, the more the overlapped regions would be. Therefore, the more messages will be flooded [15]. Using the theorem 4 in reference [15]: The number of regions generated within the sensing range of sensor $s_{i}$ witch overlaps with a group of sensors $G^{k}$ can be calculated by solving the recursive relation

$$
\begin{gathered}
Q_{n}\left(s_{i}\right)=\left\{\begin{array}{l}
f(n)=n+f(n-1)-1 \\
n \geq 1 \\
f(1)=1
\end{array}\right. \\
Q_{n}\left(s_{i}\right)=1+\frac{n(n-1)}{2}
\end{gathered}
$$


When the sensor belongs to multiple groups the number of regions can be gotten by the equation below

$$
Q^{*}\left(s_{i}\right)=\left(\sum_{\left(G_{i}^{k} \in G_{i}^{*}\right)} Q_{k}\left(s_{i}\right)\right)-\boldsymbol{\vartheta}+1
$$

where $\boldsymbol{\vartheta}$ is the number of groups.

According to [14, 15], each node in Zigzag Pattern will belongs to six sub-groups. During routing algorithm, if flooding algorithm is applied, it might consume a big amount of energy, as shown in Figure 11.

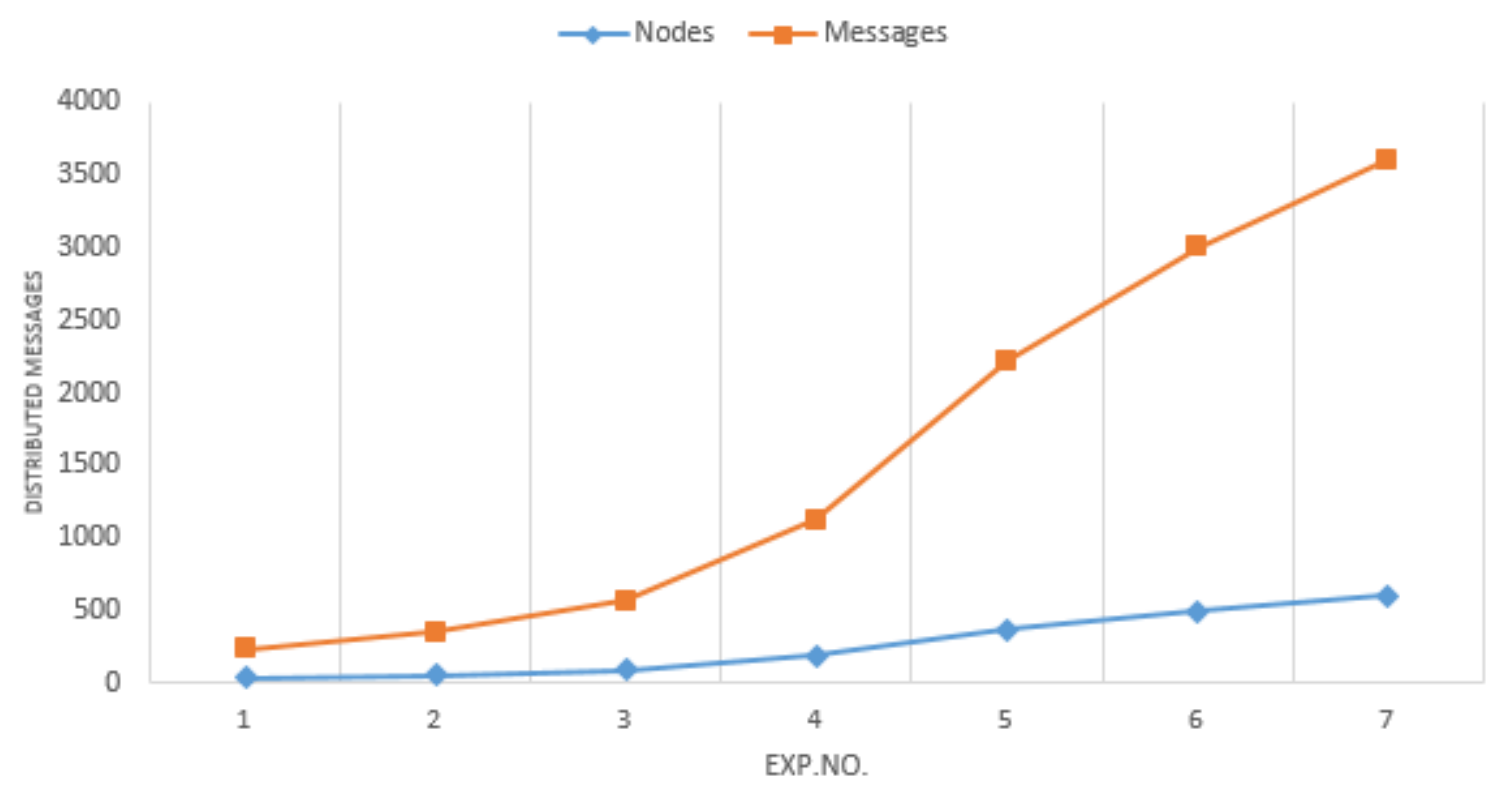

Figure 11: Flooding Messages

\section{Conclusion}

In this paper, we provided an algorithm for WSN coverage based on Zigzag Pattern. Our algorithm started by dividing the interest area into multiple Zigzag Patterns with multiple corners and lines segments. Each node is deployed in a corner of Zigzag Pattern.

Zigzag Pattern Scheme deployment algorithm expresses a very high coverage efficiency $91 \%$, as well as it expands and covers the whole interest area with minimum number of nodes, while it generates a very little coverage redundancy. In addition, we provided a geometrical analysis to clarify when the algorithm reaches the maximal and optimal coverage efficiency. The algorithm reaches the maximal and optimal coverage efficiency when the circumference of sensing range for each node is equal to the sum of its vertical Arcs length and horizontal Arcs length, while the optimal length of each line segment in Zigzag Pattern is $\approx \sqrt{3} r$ and the optimal angle of each corner is $60^{\circ}$. 


\section{1) Macrothink

Due to simplicity of Zigzag Pattern Deployment Algorithm, We will try to implement it in mobile nodes in our coming researches.

\section{Acknowledgment}

The National Natural Science Foundation of China (NO.61272472, 61232018, 61202404) and the National Science Technology Major Project (NO. 2012ZX10004301-609) support this paper. Thanks to them.

\section{Reference}

[1] A. Ghosh and S. K. Das, "Coverage and connectivity issues in wireless sensor networks: a survey,” Pervasive and Mobile Computing, vol. 4, no. 3, pp. 303-334, 2008.

[2] Waltenegus Dargie, Chistian Poellabauer “ Fundamentals Of Wireless Sensor Networks Theory And Practice " Wiley Series On Wireless Communications And Mobile Computing, 2008.

[3] Raymond Mulligan, Habib M. Ammari "Coverage In Wireless Sensor Networks: A Survey” Network Protocols And Algorithms, Vol. 2, No. 2, 2010.

[4] Aziz, N. ; Aziz, K. ; Ismail, W. (2009), 'Coverage Strategies for Wireless Sensor Networks', World Academy of Science, Engineering and Technology, International Science Index 26, 3(2), 134 - 140.

[5] Saunvit Pandya , Engel, J. ; Chen, J. ; Zhifang Fan ; Chang Liu “CORAL: Miniature Acoustic Communication Subsystem Architecture For Underwater Wireless Sensor Networks”, Irvine, CA, Sensors, 2005 IEEE.

http://dx.doi.org/10.1109/ICSENS.2005.1597661.

[6]A. Arora, P. Dutta, Et. Al, “A Line In The Sand:A Wireless Sensor Network For Target Detection, Classification, And Tracking, Computer Networks: The International Journal Of Computer And Telecommunications Networking”, Volume 46 Issue 5, 2004.

[7] H. Zhang, "Energy-Balance Heuristic Distributed Algorithm For Target Coverage In Wireless Sensor Networks With Adjustable Sensing Ranges”, Shenzhen,Information Processing, 2009. APCIP 2009. Asia-Pacific Conference on ,Volume:2 http://dx.doi.org/10.1109/APCIP.2009.247.

[8] Jie Jia, Guiyuan Zhang, Xueli Wu, Jian Chen, Xingwei Wang, and Xiaolei Yan, "On the Problem of Energy Balanced Relay Sensor Placement in Wireless Sensor Networks,” International Journal of Distributed Sensor Networks, vol. 2013, Article ID 342904, 9 pages, 2013. http://dx.doi.org/10.1155/2013/342904.

[9] A. Howard, M. J. Matari'C, And G. S. Sukhatme, “An Incremental Self Deployment Algorithm For Mobile Sensor Networks,” Autonomous Robots, Vol. 13, No. 2, Pp. 
113-126, Sep. 2002.

[10] Zou, Y.; Chakrabarty, K. Sensor deployment and target localization based on virtual forces. In Proceedings of IEEE INFOCOM, 2003; pp. 1293- 1303.

[11] Jiming Chen, Shijian Li, and Youxian Sun "Novel Deployment Schemes for Mobile Sensor Networks” Sensors 2007, 7, 2907-2919.

[12] Guodong Teng , Kougen Zheng and Wei Dong, “An Efficient and Self-Adapting Localization in Static Wireless Sensor Networks” Sensors 2009, 9, 6150-6170; http://dx.doi.org/10.3390/s90806150.

[13] ChangJun Luo1,Bo Tang ,MingTian Zhou, Zhengyin Cao “Analysis of The Wireless Sensor Networks Efficient Coverage” Chengdu, Apperceiving Computing and Intelligence Analysis (ICACIA), 2010 International Conference on. http://dx.doi.org/10.1109/ICACIA.2010.5709881.

[14] Ammar Hawbani, Wang Xingfu, Xiong Yan "Sensors Grouping Model For Wireless Sensor Network” , Journal of Sensor Technology, Vol.3 No.4 2013 "in press process".

[15] Ammar Hawbani, Wang Xingfu, Xiong Yan, Saleem Karmoshi "Wireless Sensor Network Routing Based on Sensors Grouping”, Journal of Wireless Sensor Network, Vol.6 No.1 2014." in press process".

\section{Copyright Disclaimer}

Copyright reserved by the author(s).

This article is an open-access article distributed under the terms and conditions of the Creative Commons Attribution license (http://creativecommons.org/licenses/by/3.0/). 